\title{
Lo femenino y la feminidad
}

\section{The feminine and the feminity}

\section{Carolina Rovere}

\section{RESUMEN}

Es este escrito me interesa plantear en qué puntos lo femenino y la feminidad se unen y se separan en psicoanálisis. Parto de los desarrollos de Freud, para quien estos conceptos transitan juntos, para luego ubicar qué sucede en Lacan. Al comienzo de su enseñanza y cuando sigue la posta de su maestro, femenino y feminidad van de la mano, pero en su última enseñanza son términos disyuntos. Lo femenino tiene que ver con un lugar concernido por el vacío, la ausencia de referente y lo ilimitado. Finalizo con un testimonio de final de análisis que da cuenta de la salida por lo femenino.

\section{Palabras clave}

Femenino - Feminidad - Histeria -

No-todo - Final de análisis

\section{Abstract}

It is this writing that interests me to ask in which points the feminine and femininity are united and separated in psychoanalysis. I start from the developments of Freud, for whom these concepts go together, to then locate what happens in Lacan. At the beginning of his teaching and when he follows the post of his teacher, feminine and femininity go hand in hand, but in his last teaching they are disjoint terms. The feminine has to do with a place concerned by the hole, the absence of referent and the unlimited. I conclude with a testimony at the end of the analysis that shows how the finalization is for the feminine.

\section{KEYWORDS}

Feminine - Femininity - Hysteria -

Non-whole - End of analysis 



\section{INTRODUCCIÓN}

Femenino y feminidad son dos conceptos que pueden estar juntos o disyuntos teniendo en cuenta la obra de Lacan y sobre todo lo que postula en su última enseñanza. Para introducirlo rápidamente, podemos decir que la feminidad concierne a la posición femenina y lo femenino lo excede. Nos remite a un lugar: No-todo y a un goce: goce femenino u Otro goce.

Propongo comenzar este recorrido partiendo de Freud, quien no aborda específicamente esta distinción pero sí tiene mucho para decirnos y es nuestro punto de partida.

\section{FREUD}

En los textos clásicos sobre el complejo de Edipo: "La organización genital infantil" (1923), El sepultamiento del complejo de Edipo" (1924) y "Algunas consecuencias psíquicas de la diferencia sexual anatómica” (1925), podemos extraer que para Freud la posición femenina está ligada a la lógica falo-castración. Implica un reconocimiento de la castración en la mujer, para salir a buscar un hijo del padre, como sustituto del falo que no tiene, entendiendo así la ecuación simbólica. La feminidad está íntimamente ligada a la maternidad.

No obstante, quiero establecer una diferencia con los escritos más tardíos, que no dejan hoy de sorprendernos: "Sobre la sexualidad femenina" (1931) y la conferencia "La feminidad" (1932). Estos textos nos acercan a una riquísima enseñanza, muy vigente en nuestros días y sobre todo como antesala a las Fórmulas de la Sexuación de Lacan por la disyunción entre lo femenino (No-todo) y lo paterno (Todo). En el año 1931 le da un lugar primordial a la ligazón madre preedípica y dice:

Habíamos subestimado también la duración de esta relación...Más aún: era preciso admitir la posibilidad de que cierto número de personas del sexo femenino permanecieran atascadas en la ligazón madre originaria y nunca produjeran una vuelta cabal hacia el varón... La prehistoria preedípica en la niña tiene el efecto de una sorpresa semejante a la que en otro campo produjo el descubrimiento de la cultura minoico-micénica tras la griega...En este ámbito de la primera ligazónmadre todo me parece tan difícil de asir analíticamente, tan antiguo, vagaroso, apenas reanimable, como si hubiera sucumbido a una represión particularmente despiadada. (Freud, 1931, pág. 228).

Vemos aquí que Freud plantea un atascamiento en un momento anterior al Edipo en la mujer, es decir anterior a la ley del padre, al límite que instaura esa ley. Inclusive agrega que “... la fase de la ligazón madre deja conjeturar un nexo particularmente íntimo con la etiología de la histeria (Freud, 1931, p.229)

Siempre asociamos la histeria con el amor reprimido al padre, pero Freud en 1931 nos señala que este lazo a la madre es también fundamental en la histeria, entonces la histeria se incubaría en un tiempo anterior al Complejo de Edipo.

$\mathrm{Al}$ año siguiente en la conferencia " $\mathrm{La}$ feminidad" plantea una propuesta que 
ha dejado marcas indelebles, e incluso ha tenido importantes efectos en otras teorías posteriores:

El psicoanálisis, por su particular naturaleza, no pretende describir qué es la mujer - una tarea de solución casi imposible para él-, sino indagar cómo deviene, como se desarrolla la mujer a partir del niño de disposición bisexual. (Freud, 1932, p.108)

En el año 1949 Simone de Beauvoir escribía Segundo Sexo, el segundo capítulo está dedicado a Freud, pero principalmente para criticarlo:

Los dos reproches esenciales que pueden hacerse a esta descripción provienen del hecho de que Freud la calcó sobre un modelo masculino. Supone que la mujer se siente hombre mutilado: pero la idea de mutilación implica una comparación y una valoración; muchos psicoanalistas admiten hoy que la niña echa de menos el pene, sin suponer, no obstante que la han despojado de él. (Beauvoir, 1949, pág. 46)

Pero, por otra parte Simone propone su frase célebre: "uno no nace mujer, sino que se hace". Si leemos a Freud, nos encontramos con que esta afirmación guarda la misma lógica de la citada en La feminidad. ¿Se habría servido de él?

Judith Butler en una entrevista para un documental sobre Segundo Sexo dice que cuando leyó el aforismo de Beauvoir: "no se nace mujer, sino que se hace" le produjo una gran conmoción que fue disparador para su teoría: lejos de la anatomía, el sexo es una construcción.

\section{LACAN}

En Lacan podemos ubicar principalmente tres momentos para lo femenino $y$ la feminidad: Comenzamos con la Significación del falo, allí sigue la posta freudiana pero agrega una dimensión propia en relación con la incidencia del falo en la comedia de los sexos. En estos tiempos feminidad y femenino marchan por un mismo camino. Luego se produce una torsión al inventar el objeto a en el Seminario 10. Pero el concepto de femenino se despega del de feminidad recién en los años setenta, a partir del Seminario 19.

A la altura de la Significación del falo, lo femenino está ligado a la posición femenina y, como dijimos, es el tiempo en que los conceptos transitan juntos. Lacan plantea que el encuentro entre los sexos gira alrededor de un tener y un ser con la intervención de un parecer, por eso habla de impostura y mascarada, la impostura de tener y la mascarada de ser el falo. "Es por lo que no es que pretende ser deseada al mismo tiempo que amada" (Lacan, 1958, pág. 674). Entonces, la posición femenina implica hacer de mascarada de falo, en tanto es la razón del deseo.

Un poco más tarde en el escrito "Ideas directivas para un congreso sobre la sexualidad femenina" Lacan propone lo siguiente: "el hombre sirve de relevo para que la mujer se convierta en ese Otro para sí misma como lo es para él” (Lacan, 1960, p. 711). Aquí la posición femenina implicaría encarnar esa Otra para sí misma, es decir cada mujer tendría que inventar su propia versión de lo femenino y esto se vería facilitado por la intervención de un hombre. Tenemos un anticipo de lo que 
trabajará en los Matemas de la Sexuación: lo femenino en tanto Otro, héteros.

A partir de la formulación del objeto a formalizado en el Seminario 10, lo femenino estará concernido a encarnar el semblante de ese objeto que causa el deseo. Es muy importante en este punto subrayar qué dice Lacan en relación con la angustia y la posición femenina en este seminario. Ya que aclara que las mujeres tienen una gran dificultad de ubicarse en ese lugar: "Cuando una mujer siente que es verdaderamente el objeto en el centro de un deseo,..., de esto es de lo que en verdad huye" ( Lacan, 1962- 63, p. 210) Por eso Eric Laurent, tomando esta perspectiva en El reverso de la biopolítica, afirma que "un verdadero deseo de hombre angustia al sujeto femenino" (Laurent, 2016, p. 71) Si le damos la importancia que estas citas se merecen, Lacan nos está hablando de una dificultad estructural para las mujeres, es decir no va de suyo que ser y sentirse mujer implique poder ubicarse fácilmente como causa de deseo de un hombre, porque esto supone, tal como prosigue Laurent, un llamado a suplir la falta de ese hombre, sin ninguna mediación. (Laurent, 2016, p.71). A partir de estas consideraciones podríamos pensar que cada vez que una mujer se ve convocada a recubrir la falta de cualquier Otro se angustia y retrocede. Algo muy importante se desprende de esta situación, hay que distinguir entre presentificar el objeto para alguien y hacer semblante de ese objeto a. La primer salida conduce al sufrimiento, la segunda es una solución que puede resultar interesante.

Si seguimos avanzando en su obra, nos encontramos con algo absolutamente transformador: La formalización de los
Matemas de la Sexuación que plasma en el Seminario 20 (1972-73) pero que ya había comenzado a desplegar en el Seminario 19 (1971-72) y el El Atolondradicho (14 de julio de 1972).

El giro radical que establece Lacan con las Fórmulas de la Sexuación es la separación de lo femenino con la feminidad. Lo femenino es un espacio que tiene tres atributos: el vacío, la ausencia de referente y un goce que excede al límite o ilimitado, formalizado por Lacan con los conjuntos abiertos matemáticos. ¿Cuál es la gran contribución? Que en este sitio no hay Otro, no hay referente como en el lugar Todo y por lo tanto no hay quien pueda decir qué es ser una mujer. La lógica de lo universal queda del lado Todo, junto con la perspectiva de la neurosis como estructura, la falta, el deseo, el complejo de Edipo, y por supuesto el Padre. El agregado del Notodo, es más allá del padre y sin universal. Además, lo novedoso de las fórmulas es que se sale del binarismo falo- castración para pasar a otro terreno que no plantea un orden simbólico, el padre declina y solo podemos hablar del Uno, Uno, Uno. Por eso el goce a partir de la lógica de las Fórmulas es diferente, ya no totalmente falocéntrico.

Veamos esta cita del 72 en L'Etourdit: "Lo que se llama sexo (y eventualmente el segundo, cuando es una necia) es propiamente por sostenerse de No-toda, el héteros que no puede saciarse de universo" (Lacan, 1972, p.491). No sabemos si Lacan aquí se refiere a Beauvoir, al hablar del "segundo", pero sí da el estatuto de lo femenino a lo heterosexual, al héteros o lo diferente a lo fálico. Cito: "Llamemos heterosexual, por definición, a lo que ama 
a las mujeres, cualquiera que sea su propio sexo” (Lacan, 1972, pág.491)

\section{Goce FEMENINO}

En las Fórmulas de la Sexuación tenemos dos columnas y dos pisos. La columna izquierda, lugar Todo, es el universo de la Neurosis que se funda a partir de una excepción: el Padre. Y la columna derecha o sitio del No-todo, es el territorio en donde no hay límite y Lacan llama lo femenino. Por otra parte en el piso inferior tenemos los goces: el goce fálico que corresponde al lugar Todo y el Otro goce o goce femenino que es No-todo(fálico).

\begin{tabular}{|c|c|c|c|}
\hline $\begin{array}{l}\text { B } x \\
\forall x\end{array}$ & $\begin{array}{l}\overline{\Phi x} \\
\Phi x\end{array}$ & $\frac{\overline{\frac{3 x}{V x}}}{\overline{V x}}$ & $\begin{array}{l}\overline{\Phi x} \\
\Phi x\end{array}$ \\
\hline \multicolumn{2}{|c|}{$\alpha$} & \multicolumn{2}{|l|}{$S(X)$} \\
\hline & & & \\
\hline
\end{tabular}

Es interesante observar los detalles que tiene esta escritura ya que el goce de La mujer tiene una línea que apunta al goce femenino, en estos tiempos escrito como $\mathrm{S}(\mathrm{A})$, y otra que se dirige al goce fálico $\phi$.

Veamos estas dos referencias de Lacan:

"La mujer con respecto a la función fálica, se sitúa por estar no toda sujeta a ella" (Lacan, 1971-72, pág.100) y luego "La mujer es no toda porque su goce es dual” (Lacan, 1971-72, pág. 101)

En la propuesta de Caras del goce femenino aparece de esta manera:

En el caso del goce femenino, tal como lo podemos leer en la imagen, se puede ver que estas líneas que remiten a la mujer a dos tipos de goces, son móviles...El goce en la mujer no es algo ni rígido ni estable. Cada situación de vida la convoca a una modalidad diferente de gozar. Lo importante es que los goces se articulan, como se muestra en el esquema de Lacan, las piernas están imbricadas (Rovere, 2011, p. 45)

Pero, ¿por qué no pensar hoy que es una sola línea? Lacan no divide a esa recta, solo la dobla, y le coloca dos flechas en cada punta. Esto no es una casualidad, si sostenemos que es una única línea podemos dilucidar que existe un mismo cursor para los dos goces, una recta que se dobla y hace vértice en Ła. Entonces habría una conexión entre el goce femenino y el fálico, pero no como efecto de una imbricación, sino como un movimiento topológico continuo. Así lo indica la recta y sus dos puntas que se orientan por dos modalidades diferentes de gozar. Esto concierne tanto a los hombres como a las mujeres. Dicho de una manera más acorde a la época actual: al parlêtre, concepto cosechado de la última enseñanza de Lacan.

Entonces como vemos, lo femenino ya no tiene mucho que ver ni con la feminidad, ni con las mujeres, ni con el binarismo. Esto es lo disyunto de la enseñanza tardía de Lacan.

\section{HISTERIA Y HOMOSEXUALIDAD}

Sabemos que la histeria está habitada mayoritariamente por mujeres por una cuestión de estructura. Es una clínica que se orienta por el amor reprimido al Padre, 
el deseo sostenido en la insatisfacción y la pregunta: ¿cómo goza una mujer?, que es también inconciente y por lo tanto la podemos rastrear en sus formaciones. Esta pregunta se formula en tanto modo de vivir la neurosis porque el goce femenino es enigmático, por más que se haya intentado desde muchos frentes encontrar una evidencia clara que tranquilice a las ciencias.

Lacan a partir de las escrituras sexuales dirá que "la histeria es hacer de hombre y ser por lo tanto homosexual" (Lacan, 1972-73, p.103) Es muy importante esta cita del Seminario Aún porque nos permite entender que hacer de hombre es habitar el lado homo, el lado para todos fálico y la histérica es fálica por estar en posición viril.

Entonces la histeria está en el lado Todo de las Fórmulas de la Sexuación, allí donde hay un referente que puede ser el Padre y/ o la verdadera mujer o LA mujer sin barrar. Por eso necesita crearse una ficción que es una Otra mujer que ella cree que sabe realmente de qué se trata lo femenino. Alguien que encarne su pregunta, tal como es la Sra. K para Dora.

La lucidez de Marie-Hélène Brousse nos permite ubicar qué sucede hoy con la posición histérica en los tiempos de la caída del padre:

La posición histérica ya no requiere pasar por el padre y los hombres para tener acceso a lo femenino... sería lógico ver a las Doras de hoy pasar de una posición homosexual inconciente,.. a una posición consciente y a un acting out de la atracción por lo femenino en la otra mujer (Brousse, 2013, p. 28-29)
El planteo de Brousse, orientado por su clínica nos permite pensar la particularidad de la histeria y su relación a la homosexualidad en nuestro tiempo. Es decir en la clínica nos encontramos con homosexualidades femeninas como modo de responder al enigma de lo femenino de una manera directa y sin mediación con el hombre.

\section{LO FEMENINO AL FINAL DE UN RECORRIDO DE ANÁLISIS}

La finalización de un análisis concierne a lo femenino, porque consiste en ir más allá del padre y de los referentes fijos que conforman la neurosis de cada quien. Implica vincularse con el vacío que Lacan escribe $\mathrm{S}(\mathrm{A})$ en las fórmulas. Salir de un análisis es obtener alguna solución con lo que no hay y esto conlleva una nueva escritura que es contingente. La contingencia es una escritura que surge como efecto de lo imposible. Es decir: en el lado femenino, las dos escrituras del piso de arriba conciernen a las categorías de imposible primero y contingente después. La contingencia es entonces una escritura efecto de una no escritura.

Como modo de ejemplificar la salida de un análisis por lo femenino, me interesa extraer un último sueño relatado por Débora Rabinovich ${ }^{1}$ en uno de sus testimonios.

"En la sala de espera del analista solo somos dos personas. Una mujer y yo. La conozco. Representa para mí esa Otra mujer desencadenante de la separación con mi marido. Es morena, alta, extranjera, y con un italiano perfecto. Sobre la mesa, en el lugar donde siempre está el florero 
con un lindo ramo de flores, hay un teléfono. Ella me pregunta, en francés, mientras lo señala, cómo se llama esa parte del teléfono. Miro, pienso y digo no sé. Y no lo sé en ningún idioma. Me despierto muy tranquila. ¡Ni ella, ni yo, y en ningún idioma!” (Rabinovich, 2016, p. 49)

El sueño nos demuestra que no hay nombre para lo femenino, que tampoco hay LA mujer que sabe sobre lo femenino, que solo hay Una mujer y Una mujer porque nadie sabe qué es ser mujer. Se trata, tal vez de que cada Una invente algo interesante con el vacío que habita el cuerpo femenino.

\section{NOTAS AMPLIATORIAS}

1 Débora Rabinovich fue AE (analista de la Escuela ) de la AMP (Asociación Mundial del psicoanálisis) durante el período 2013-2017. Esta nominación se obtiene a partir de pasar por el dispositivo del pase que inventó Lacan por el cual un sujeto, que considere haber finalizado su análisis, decide testimoniar por su recorrido a los fines de la transmisión a la comunidad analítica, tomándose a sí mismo como caso clínico.

\section{REFERENCIAS BiBLIOGRÁFICAS}

Beauvoir, S. (1949) El segundo Sexo, Buenos Aires, Argentina: Debolsillo, 2012.

Brousse, M-H. (2013) L'homosexualité féminine au pluriel ou quand les hystériques se passent de leures hommes de paille" en Harrison, S. Elles ont choisi, Paris, Francia: Michèle

Butler, J. Entrevista en: Simone de Beauvoir - No Se Nace Mujer (Documental), recuperado de https:// youtu.be/WiNF3At3XFk

Freud, S. (1923). "La organización genital infantil", en Obras completas, Buenos Aires, Argentina: Amorrortu.

(1925). "Algunas consecuencias psíquicas de la diferencia sexual anatómica", en Obras completas, Buenos Aires, Argentina: Amorrortu.

(1931) "La feminidad", en Obras Completas T. XXII, Buenos Aires, Argentina: Amorrortu.

(1932). "Sobre la sexualidad femenina", en Obras Completas, Tomo XXI, Buenos Aires, Argentina: Amorrortu.

LACAN, J. (1958) "La significación del falo", en Escritos II, Buenos Aires, Argentina: Siglo XXI.

(1960) "Ideas directivas para un congreso sobre la sexualidad femenina", en Escritos 2, Buenos Aires, Argentina: Siglo XXI.

(1962-63). El Seminario, Libro 10. La Angustia. Clase del 20 de marzo de 1963, Buenos Aires, Argentina: Paidós. (1971-72). El Seminario: Libro 19,... Ou pire, Buenos Aires, Argentina: Paidós. 
(1972). El Atolondradicho, en

Otros Escritos, Buenos Aires, Argentina: Paidós.

(1972-73). El Seminario: Libro 20, Aun, Buenos Aires, Argentina: Paidós. Laurent, E. (1999) Posiciones femeninas del ser, Buenos Aires, Argentina: Tres Haches.

LAURENT, E. (2016) El reverso de la biopolítica, Buenos Aires, Argentina: Grama.

Rabinovich, Débora. (2016). Teléfono. Revista Lacaniana de Psicoanálisis, volumen (20), 43-49, Buenos Aires, Argentina: EOL.

Rovere, C. (2011). Caras del goce femenino, Buenos Aires, Argentina: Letra Viva.

COMO CITAR ESTE ARTículo:

Rovere, C (2019). Lo femenino y la feminidad en Revista Psicoanálisis en la Universidad $N^{\circ} 3$. Rosario, Argentina. UNR Editora.

Carolina Rovere

Psicóloga , Psicoanalista. Magíster en Psicoanálisis. Docente e investigadora de la Facultad de Psicología de la UNR. Miembro de la EOL (Escuela de la Orientación Lacaniana) y de la AMP (Asociación Mundial del Psicoanálisis). Coordinadora del área de Psicología de CERIN Rosario(Centro de Rehabilitación infantil). Autora del libro Caras del goce femenino (2011) Letra Viva y La palabra que falta es Una mujer en co-autoría con Sergio Zabalza (2013) Letra Viva. 